\title{
A 67-year-old man with anterior neck swelling
}

\author{
Kathryn Volz $\cdot$ Carlo Rosen · Richard Wolfe • \\ Kevin Ban · John Sakles · Kenny Bramwell · \\ Danny Davis · Peter Rosen $\cdot$ Leon Sanchez
}

Received: 27 April 2009/Accepted: 5 May 2009/Published online: 17 June 2009

(c) SIMI 2009

Dr. Carlo Rosen: The patient was a 67-year-old man who just got off an airplane from Florida complaining of anterior neck swelling that developed either late the night prior or early that morning. He was concerned enough in Florida that he wanted to come to Boston, where all his doctors were, so he obtained a flight to Boston with his wife. He said over the past few hours, the swelling had gotten worse, and that he had developed a little bit of a hoarse voice. Initially he got put into one of our resuscitation rooms, and a quick history revealed that he was on coumadin for atrial fibrillation. He also denied fevers, denied any history of anything like this happening in the past, denied rash or any pain or discomfort anywhere else, and was able to give a full history by himself. Other past medical history was significant for a previous carotid stent and asthma. He did say he had allergies to shellfish, associated with a rash, and took no other medications but the coumadin. Are there any questions?

K. Volz $(\varangle) \cdot$ C. Rosen · R. Wolfe - K. Ban · P. Rosen ·

L. Sanchez

Department of Emergency Medicine, Beth Israel Deaconess

Medical Center, One Deaconess Road, WCC2, Boston,

MA 02215, USA

e-mail:kvolz@bidmc.harvard.edu

J. Sakles

Department of Emergency Medicine, University of Arizona,

Tucson, AZ, USA

K. Bramwell

Emergency Medicine of Idaho, St. Luke's Regional Medical Center, Boise, ID, USA

D. Davis

Department of Emergency Medicine, University of California San Diego Medical Center, San Diego, CA, USA
Dr. Richard Wolfe: How many hours passed from the onset of his symptoms to the point he presented?

Dr. C. Rosen: He was vague about the exact timing. He probably had symptoms the night before starting with the swelling, and the voice change happened over the past few hours.

Dr. Wolfe: You saw him roughly at what time?

Dr. C. Rosen: Probably early afternoon. He took a taxi from the airport to the Emergency Department (ED).

Dr. C. Rosen: To me there were two worrisome parts to the history. One was the swelling which had gone on for probably $18 \mathrm{~h}$. The second, and more concerning, was the voice change, which he said had occurred over 2-3 h.

Dr. Wolfe: I am not clear why he was taking coumadin. There was absolutely no history of any trauma, correct?

Dr. C. Rosen: There was no history of trauma. He was on coumadin for atrial fibrillation, and said he had been taking his usual dose. On physical examination the vitals were: temperature of $36.5^{\circ} \mathrm{C}\left(97.7^{\circ} \mathrm{F}\right.$, ) pulse 117 beats per minute, blood pressure 156/88 torr., respirations 25 breaths per minute, and oxygen saturation $99 \%$ on room air. $\mathrm{He}$ looked very comfortable. The only alarming thing about the examination was he had a slightly hoarse voice. I think we picked up the voice changes more than he himself or his wife did. He clearly had anterior submandibular neck swelling from the whole anterior mid to upper part of the neck. He had some tongue elevation and some sublingual ecchymosis; it was not woody, but it was definitely feeling full. The rest of the examination was normal. He was totally appropriate and wide awake and not in a whole lot of distress. 
Dr. Wolfe: The differential here is either infectious or a hematoma. It is possible that he has a Ludwig's angina, although you are not describing a lot of pain, fever, or symptoms suggestive of that. Even though it's a much more unusual entity, submandibular hematoma secondary to being on coumadin is what this is sounding like with the ecchymosis and the slow progression of worsening signs of deterioration. The importance of the latter diagnosis is if you are betting on hypercoagulation and subsequent bleeding, you want to rapidly reverse anticoagulation. Then you have the million dollar question we are here for, does he need his airway actively managed or not? I think with the vocal change and deterioration my assumption is that you felt that he did need his airway protected. We might argue that you have time since the progression has been slow. If you start to reverse him, could you put him in an area where you could watch him closely, and do you have enough time to reverse the prothrombin time towards normal before you have to launch into a difficult airway? Nevertheless, with a respiratory rate of 25 breaths a minute, with the vocal changes, and with the time frame it would take for him to turn around, waiting for reversal means that the airway is likely to be more difficult to intubate, therefore my preference would be to get the airway controlled right away.

Dr. C. Rosen: I was thinking he needed his airway managed, but he didn't need his airway managed this very minute. I thought we had a little bit of time to plan, but I wasn't going to let this guy sit much longer. The thing that made me nervous, and encouraged me to make that decision was really the voice change over a couple of hours.

Dr. Kevin Ban: This is the moment that defines the emergency medicine specialist. Often we are asked to treat or stabilize a patient's symptoms before we identify the precise medical condition causing these symptoms. Dr. Wolfe correctly identifies an expanding hematoma as one possible etiology of the patient's symptoms, but we must be sure to consider other disease entities like infection. Initially, we must focus upon stabilizing this patient's airway so that we have time to identify and treat the underlying cause of his pending airway instability. If one delays managing his airway, it may prove to be much more difficult as the patient worsens.

Dr. John Sakles: I think it is pretty straightforward. I don't think you have more room to wait here. You have a guy who is anticoagulated, probably has a supra-therapeutic INR, with progressive throat swelling, tongue swelling, and difficultly swallowing. Moreover, now he has changes in his voice. In my mind there aren't a lot of additional clinical characteristics that you have left that you could observe that would change the decision to intubate. He is already demonstrating signs of impending airway obstruction. I would act now rather than wait for things to worsen. If you observe this patient much longer, you won't be able to perceive further clinical deterioration in a reasonable time frame. The next thing you will see is complete airway obstruction; and then it's too late.

Dr. Ban: If this is an expanding hematoma, the more time you wait, the more likely the anatomy will become progressively more distorted making early airway management mandatory.

Dr. Kenny Bramwell: In a way this is similar to an inhalational burn victim. The difficult thing is to act aggressively when the patient still has the appearance of doing reasonably well.

Dr. Leon Sanchez: I think we are all fairly sure this is due to coumadin toxicity. We need to get a few of the other things ordered that are going to help fix the problem such as fresh frozen plasma (FFP), vitamin K, and Factor IX Complex if available or used in the institution. Those things can be done in parallel so that once you have the airway under control you can actually start to treat the underlying condition.

Dr. C. Rosen: We felt that we had time to treat with Factor IX Complex first. We obviously weren't going to wait for the Factor IX Complex to kick in, but wanted it on board in case things got ugly. We actually gave the Factor IX Complex initially the minute he was in the resuscitation room, and then the vitamin $\mathrm{K}$, and the FFP was thawing.

Dr. Ban: This makes good sense since your backup rescue airway might be surgical if orotracheal intubation is impossible. Did you set up for an emergent airway while you were reversing the patient?

Dr. C. Rosen: He arrived in the room at 8:05 and got Factor IX Complex at 8:05. My thought initially was I did not want to paralyze this patient. On a little more careful examination he had a hematoma pushing the trachea over a little bit at that point. My plan was initially to follow the airway protocol that we have for identifying a difficult airway, and use a triple team approach. We called anesthesia to bring down their fiberoptic scope. I think that is something that's worthwhile, although obviously from a systems issue not every place is going to have all these resources available. Our institutional airway protocol for a difficult airway includes calling anesthesia for backup and equipment if you identify a difficult airway. This is one scenario where I thought involving ENT right up front was also helpful, and that's what we did as well. The plan was to initiate fiberoptic airway management without actually paralyzing him and without even taking a look orally with the laryngoscope. I thought he was going to have the 
potential for anatomical distortion, and I just did not want to mess around with the laryngoscope. We had the resources of the fiberoptic scope available, so the plan was to proceed directly with fiberoptic visualization.

Dr. Wolfe: I think what makes the case interesting is it's clearly a difficult airway, but mainly we have a relative contraindication to cricothyrotomy in the patient's coagulopathy, and the fact that there is a large tense hematoma right there makes people nervous. In fact, if you really are pushed to a surgical airway when you open up the neck at least one benefit is you will decompress the hematoma, and actually make the distortion less, and potentially even allow you to be able to ventilate the patient more effectively. So the contraindication is relative but not absolute, but it is prudent to have back up. I would think that if you do proceed to a surgical airway you can anticipate a lot of bleeding and difficulty with control, therefore having ENT there is absolutely a prudent approach, both from the standpoint of helping with the surgical airway, but occasionally for assistance even with the endoscopic component.

Dr. Ban: In a tertiary care hospital when you have multiple levels of back up with subspecialists, this is the correct approach. When consultants are not immediately available, what is the best approach to manage this patient's airway?

Dr. C. Rosen: I think that is a tough question. Thinking through this case after the fact, if you were in the community, you probably are not going to have enough time to transfer a patient to a place that has all those resources. I would probably do some form of oral awake intubation, without paralyzing the patient, have equipment ready and a general surgeon who would be able to help you do a cricothyrotomy, to have another set of hands there if you need to proceed to a surgical airway.

Dr. Sakles: I think that for the community physician, awake fiberoptic intubation is probably not going to be practical. Although that is probably the ideal approach, the average emergency physician is just not going to be skilled enough to do a fiberoptic intubation in a scenario like this. I think the best option is probably going to be using a rigid video laryngoscope. Using a conventional laryngoscope and trying to compress the tongue is likely to be impossible. The tongue is going to be rock hard, and there will be nowhere to displace it. The nice thing about the video laryngoscope is that you can put it in there, and essentially see around the back of the tongue without having to compress it. I think with the less experienced airway manager, the rigid video laryngoscope is really the best way to go, and then if that fails I think a surgical airway is probably the next option.
Dr. Wolfe: If they have a rigid scope that approach is good, but a lot of physicians will be in EDs where they just have the basic stuff, they might have a bougie, maybe an LMA. There could be an argument made that you go with what you know best, perform a rapid sequence airway, and give yourself the one best shot, because the patient is stable and you can preoxygenate to buy a little time. Then you are just prepared to move rapidly to surgical airway if there is any difficulty.

Dr. Sakles: I think that's reasonable if that's the only thing you have available. Nevertheless, I think the likelihood of direct laryngoscopy being successful would be extremely low in a situation like this. It really is going to be hard to get the tongue out of the way.

Dr. Wolfe: I actually pulled the very limited literature on this, there may be about 12 cases reported of similar hematomas with potential airway issues [1-10]. In the reports, a variety of approaches were used including endotracheal intubation $[2,5]$. But I agree with you, I would suspect that you are going to be in trouble trying direct laryngoscopy, however you will only know when you are there. My problem with an initial direct oral airway approach is that you may create difficulties on top of the ones the existing the disease is producing, such as bleeding or laryngospasm, and so you may reduce the chance of securing the airway without a surgical approach.

Dr. Sanchez: The one thing I have done in the past when I have thought there might be a difficult airway, but still want to do rapid sequence intubation (RSI), is to start with the bougie. I start with the bougie because if you see the airway you can pass it through the airway, if you can't see the airway, then you can still use the bougie by feel [11]. This way you are using only one tool, and you will save time if you can't get a good look to pass the endotracheal tube.

Dr. Ban: I agree with this approach, and I think that while it makes sense to try to avoid paralyzing this patient, if you are able to pre-oxygenate and relax the musculature, you'll optimize your chances of getting that first pass airway if you don't have any video assisted or fiberoptic assisted airway tools. I agree with Dr. Sanchez, and would try to place a bougie into the patient's trachea, through direct visualization or by tactile feel. Once the bougie is safely in the trachea, I would pass the tube using the bougie as a guide. I would not try to just pass the tube, even with a stylet, because there may be airway distortion preventing the placement of the tube in the trachea. While I agree with Dr. Wolfe that supra-therapeutic levels of coumadin is a relative contraindication to a surgical airway, without any backup one must move expediently to the ultimate rescue technique by performing a surgical airway. The patient deserves that chance if all other attempts fail. 
Dr. Wolfe: I agree with that.

Dr. Sakles: I have one comment about the approach Dr. Wolfe was mentioning. Sometimes what I'll do to optimize the first attempt if I'm reluctant to paralyze someone, is try an initial "awake" attempt with ketamine before the RSI. I'll get both succinylcholine and ketamine ready. Then I'll push the ketamine first, maybe 50-70 mg, and then take an "awake" look at the airway. This way I haven't compromised the airway by paralyzing the patient. If I can see the airway, I go ahead and pass the tube, and if that doesn't work then I push the succinylcholine and proceed to RSI to maximize my intubation conditions. Ketamine can give you one more option before you head down the paralytic pathway. It provides excellent conditions for an awake look at the airway without causing loss of airway reflexes or respiratory depression.

Dr. Wolfe: I think that is a great maneuver.

Dr. Danny Davis: I will offer a slightly different perspective to muddy the waters a bit. It would be interesting to see how many ED's have a rigid videolaryngoscope at present-I would predict that most of these also have ENT, anesthesia, or pulmonary physicians with flexible bronchoscopy available. We have to offer an alternative for the ED without any form of indirect laryngoscopy. I would suggest that even with an expanding hematoma such as this one, direct laryngoscopy would be successful most of the time due to a combination of jaw muscle relaxation and the distensibility of tissue around the hematoma, both of which would likely allow a view of the cords. As a general rule, if the patient can open his/her mouth enough to allow visualization of the uvula while awake; I am willing to make an attempt at direct laryngoscopy following RSI if no videolaryngoscope or fiberoptic options are available.

To make the procedure safer, I am going to focus my preparatory efforts on two things. First, I am going to preoxygenate the patient to maximal levels by applying a nonrebreather mask, and having the patient take maximal tidal volume breaths for several minutes. This will increase alveolar surface area and wash out all nitrogenous air from the lungs, buying me up to five or more minutes in most cases. This is a reasonable expectation with this patient, since he can still talk, and presumably has nothing wrong with his lungs. Second, I am going to take the time to identify the relevant anatomy should cricothyrotomy become necessary. This includes preparing the neck with betadine, and using a pen to outline structures as much as possible. We have even used ultrasound to assist in this capacity. As soon as it becomes clear that visualization of glottic structures is not feasible, attempts should be abandoned and bag-valve-mask ventilation (or supraglottic airway insertion) should be initiated to maintain oxygenation during cricothyrotomy.

While performing a cricothyrotomy on a coagulopathic patient is certainly not at the top of my list of desirable airway approaches, it is critical to underscore the importance of a "tactile cricothyrotomy" in such cases. By this I mean that expecting to see the anatomy like in a textbook or cadaver lab is unrealistic, and will lead to costly delays. Instead, the left index finger should immediately be inserted into the incision once the dermis is separated, and the relevant anatomy (thyroid cartilage or "Adam's apple", cricothyroid membrane, and cricoid ring) palpated. The tip of the scalpel can even be used to "tap" down the thyroid cartilage until the trampoline-like cricothyroid membrane is reached. Once punctured, the operator should expect to witness a geyser of blood and air with each bagvalve-mask breath, confirming proper location. The tracheal hook can then be inserted down the scalpel to maintain control at the thyroid cartilage while an airway is inserted. This tactile approach can be performed even with large amounts of blood or swollen anterior neck tissue.

Dr. C. Rosen: Let me present what actually happened. Anesthesia and ENT arrived, and the anesthetist took the approach that a lot of you guys have talked about, he attempted to look with a glidescope. The reality was that the tongue was not that hard or swollen. They thought they would be able to get the tongue out of the way, and that the hematoma might be more anterior. We had a pretty brief discussion and came up with this plan with the anesthesia attending. Plan B was for ENT to proceed with a fiberoptic scope. During anesthesia's attempt, the ENT chief resident had the fiberoptic scope in her hand, and was ready to proceed immediately with that as a backup. The anesthesia attending came and gave a pretty hefty dose of propofol as they were taking a look. They started to take a look with the glidescope, but it proved to be an unsuccessful attempt to visualize anything. The oxygen saturation that had been in the high $90 \mathrm{~s}$ started to drift down to about $86 \%$.

Therefore, we went to plan B. The ENT resident took a look, and interestingly saw a lot of supraglottic edema, with pooling of secretions and a very tight airway. Nevertheless, it was possible to visualize the anatomy, suction the secretions, and then pass the fiberoptic scope into the airway. Passing the tube was not that easy, and we encountered a little bit of resistance. Obviously we were concerned about the hematoma, but at some point we were able to get the tube in through the resistance and with reasonably good visualization. After the tube was in place the oxygen saturation came up.

Dr. Wolfe: What was the subsequent course on the patient? 
Fig. 1 CT scan of neck

following intubation

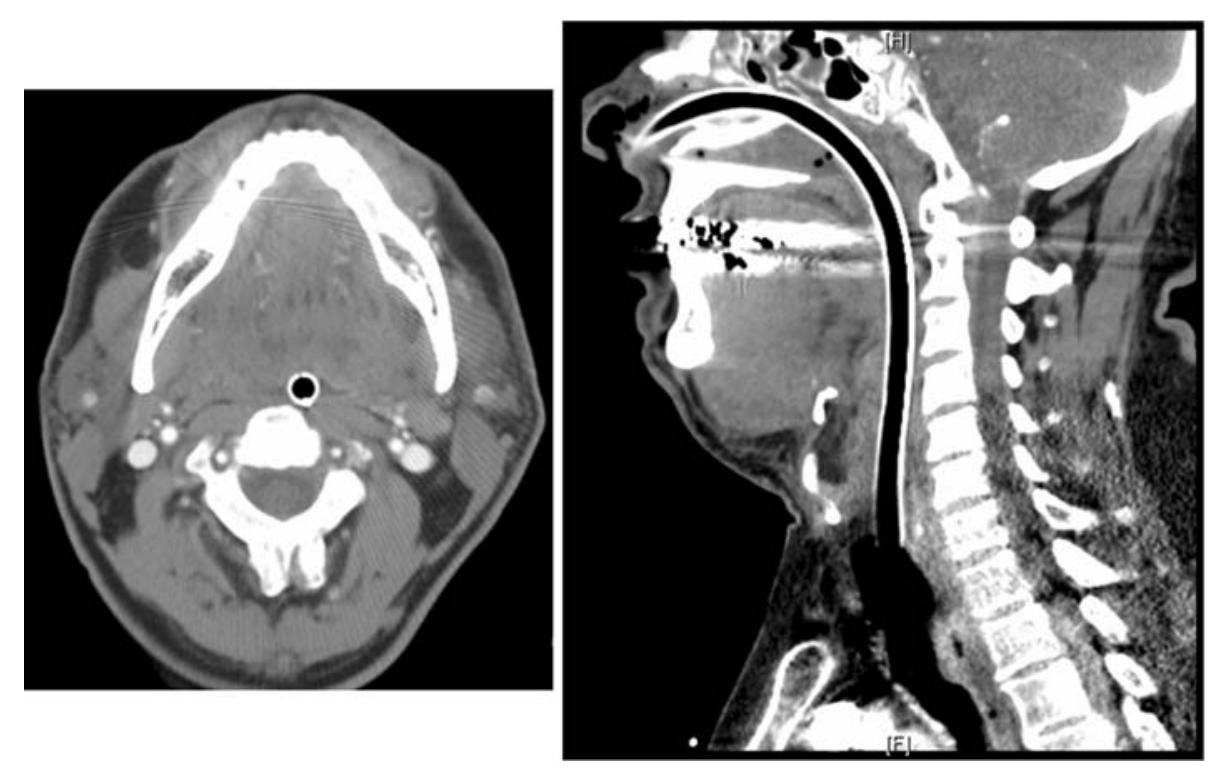

Dr C. Rosen: The subsequent course was interesting in that the CT scan didn't really show an impressive hematoma. The radiologists read the study as a very small hematoma, maybe some edema, no air fluid levels, but not a significant hematoma. Based on this read and the edema that was visualized in the airway, there was concern that maybe this was in part an allergic reaction. The reality, however, was that the INR came back at eight, and given the speed of the airway and voice changes, I think retrospectively it was a hematoma, especially with really nothing else going to support the diagnosis of an allergic reaction. That CT result was confusing. The patient was admitted to the ICU. On hospital day 6, ENT performed direct laryngoscopy on the patient, and saw subglottic swelling and a hematoma, but an open airway, and then the next day, extubated the patient (Fig. 1).

Dr. Ban: Did the patient have any reason why his INR would have been supra-therapeutic?

Dr. C. Rosen: No he did not. He was not taking any other medications, and he seemed to have been taking the coumadin as directed.

Dr. Ban: What did you treat him with in the ED prior to going to the ICU?

Dr. C. Rosen: He received profil IX immediately, and then he got vitamin $\mathrm{K}$, intravenously (IV) and FFP. We gave the vitamin $\mathrm{K}$, IV, because we saw this as life threatening bleeding requiring immediate reversal. He also got steroids, zantac, benadryl, and per ENT, empiric clindamycin as well. He got treated to cover all three potential problems on the differential.
Dr. Peter Rosen: I think this case nicely illustrates that anatomic distortion is often worse when you look at the airway than what you would predict from the patient's initial clinical appearance, and from imaging studies. It shows once again, that we don't have much leeway when we think the airway is compromised, and that the managing emergency physicians were absolutely correct to be worried about impending airway distortion.

Conflict of interest statement The authors declare that they have no conflict of interest related to the publication of this manuscript.

\section{References}

1. Boster SR, Bergin JJ (1983) Upper airway obstruction complicating warfarin therapy: with a note on reversal of warfarin toxicity. Ann Intern Med 12:711-715

2. Cohen AF, Warman SP (1989) Upper airway obstruction secondary to warfarin-induced sublingual haematoma. Arch Otolaryngol Head Neck Surg 115:718-720

3. Duong TC, Burtch G, Shatney CH (1986) Upper airway obstruction as a complication of oral anticoagulation therapy. Crit Care Med 14:830-831

4. Gupta MK, McClymont LG, Hamdy El-Hakim (2003) Case of sublingual hematoma threatening airway obstruction. Med Sci Monit 9:CS95-CS97

5. Lepore M (1976) Upper airway obstruction induced by warfarin sodium. Arch Otolaryngol Head Neck Surg 102:505-506

6. Ozpolat B, Yilmaz MA, Yücel E (2007) Lingual hematoma threatening airway obstruction in a patient on oral anticoagulation with warfarin. Blood Coagul Fibrinolysis 18:575-576

7. Lim M, Chaudhari M, Devesa P, Waddell A, Gupta D (2006) Management of upper airway obstruction secondary to warfarin therapy: the conservative approach. Department of Otolaryngology, Great Western Hospital, Swindon, UK. PMID J Laryngol Otol 120:12 
8. Murray JM, Blunnie WP (1986) Acute upper airway obstruction as a complication of oral anticoagulant therapy. Crit Care Med $14: 830-831$

9. Rosenbaum L, Thurman P, Krantz SB (1979) Upper airway obstruction as a complication of oral anticoagulant therapy: report of three cases. Arch Intern Med 139:1151-1153
10. Lai YY, Lin HJ, Te AL, Chen KT (2006) Spontaneous sublingual haematoma resulting in airway obstruction. Emerg Med J 7:583

11. Shah KH, Kwong BM, Hazan A, Newman DH, Wiener D 2008 Success of the gum elastic bougie as a rescue airway in the Emergency Department. J Emerg Med Nov 8 